\title{
Matched analysis of induction chemotherapy plus chemoradiotherapy versus induction chemotherapy plus radiotherapy alone in locoregionally advanced nasopharyngeal carcinoma: a multicenter study
}

\author{
Bin Zhang ${ }^{1,2, *}$, Ying Hu ${ }^{1, *}$, Rui-Hua Xiong ${ }^{3, *}$, Yu-Fei Pan ${ }^{4,}{ }^{*}$, Qian-Lan Xu ${ }^{1}$, Xiang-Yun \\ Kong $^{1}$, Rui Cai ${ }^{1}$, Qiu-Qiu Chen ${ }^{1}$, Hua-Ying Tang ${ }^{1}$ and Wei Jiang ${ }^{1}$ \\ ${ }^{1}$ Department of Radiation Oncology, Affiliated Hospital of Guilin Medical University, Guilin, PR China \\ 2 Department of Radiation Oncology, Wuzhou Red Cross Hospital, Wuzhou, PR China \\ ${ }^{3}$ Department of Radiation Oncology, 181st Hospital of People's Liberation Army, Guilin, PR China \\ ${ }^{4}$ Department of Radiation Oncology, Nan Xishan Hospital, Guilin, PR China \\ * These authors have contributed equally to this work \\ Correspondence to: Wei Jiang, email: weijiang@glmc.edu.cn \\ Keywords: nasopharyngeal carcinoma, induction chemotherapy, radiotherapy, concurrent chemoradiotherapy \\ Received: June 09, 2016 \\ Accepted: October 12, 2016 \\ Published: November 10, 2016
}

\section{ABSTRACT}

Background: The relative efficacy of induction chemotherapy (IC) followed by concurrent chemoradiotherapy (CCRT) versus IC followed by radiotherapy (RT) alone in locoregionally advanced NPC remains unclear.

Methods: A total of 877 patients with locally advanced NPC who underwent IC/ CCRT or IC/RT at four institutions in China between January 2004 and December 2010 were retrospectively assessed. IC was cisplatin-based combination chemotherapy; concurrent chemotherapy, single agent cisplatin. Two-dimensional conventional radiotherapy (2DCRT) was the radiotherapy technique. All patients were matched in an equal ratio using a pair-matched method. Overall survival (OS), disease-free survival (DFS), distant metastasis-free survival (DMFS), locoregional relapse-free survival (LRRFS) and toxicities were assessed.

Results: Eligible patients were matched $(n=642 ; 321$ patients per arm) based on eight clinicopathological characteristics. Five-year OS, DFS, DMFS, and LRRFS were $76 \%, 70 \%, 86 \%$, and $88 \%$ for IC/CCRT and $75 \%, 70 \%, 90 \%$, and $91 \%$ for IC/RT, respectively. There were no statistically significant survival differences between arms $(P>0.05)$, even in subgroup analysis. In multivariate analysis, treatment (IC/CCRT vs. IC/RT) was not an independent prognostic factor for any survival end-point. Grade $3 / 4$ acute gastrointestinal toxicities (vomiting, nausea) and hematological toxicities (leucopenia/neutropenia, thrombocytopenia and anemia) were significantly more common in the IC/CCRT arm than IC/RT arm during RT.

Conclusion: Overall, IC/CCRT failed to demonstrate any survival advantage but higher acute toxicities over IC/RT in locoregionally advanced NPC.

\section{INTRODUCTION}

Nasopharyngeal carcinoma (NPC) is an endemic malignant tumor that is highly prevalent in a number of regions, including southeast Asia and southern China [1]. As it is highly chemo- and radiosensitive, chemotherapy in combination with radiotherapy (RT) has become the standard recommended treatment in the NCCN guidelines for locoregionally advanced NPC [2].

Numerous studies have demonstrated that induction chemotherapy (IC) followed by concurrent chemoradiotherapy (CCRT) represents an effective approach to significantly improve survival in locoregionally advanced NPC [3,4]. This strategy is 
widely applied in the areas of China where NPC is endemic. However, a high frequency of toxicities is observed during concurrent chemotherapy and 20\%-40\% of patients with NPC cannot complete their planned course of concurrent chemotherapy due to severe toxicities $[5,6]$. Therefore, the optimal sequence of RT and chemotherapy in NPC remains to be clarified.

As far as we are aware, it is unclear whether the addition of concurrent chemotherapy to RT administered after IC improves the efficacy of treatment compared to IC/RT. Encouraging results for IC plus RT alone have been reported in clinical studies. Zeng et al. reported IC/ RT provided good survival outcomes, with 5-year overall survival, cancer-specific survival, distant metastasisfree survival, and locoregional relapse-free survival rates of $62.1 \%, 65.2 \%, 88.2 .4 \%$, and $75.3 \%$ [7]. Wu et al. reported an equivalent 3 -year overall survival rate of $86.8 \%$ for IC/RT, which was similar to the rate observed for CCRT in the same study [8]. In particular, as imaging techniques have developed, magnetic resonance imaging (MRI), 18F-fluorodeoxyglucose (FDG) positron emission tomography/computed tomography, (18F-FDG PET/CT) and high-resolution $\mathrm{CT}(\mathrm{HRCT})$ can provide more accurate target imaging, which has immensely improved the efficacy of radiotherapy alone in NPC $[9,10]$. It should be noted that similar survival outcomes were documented for the IC/RT and IC/CCRT arms in a small retrospective trial [11]. Therefore, the evidence in the literature suggests that IC followed by RT alone appears to be a feasible treatment strategy for locoregionally NPC.

At present, no high-quality randomised clinical trials have compared the efficacy of IC/RT and IC/ CCRT in locoregionally advanced NPC, it is necessary to compare the efficacy and toxicities of IC followed by CCRT or RT alone in larger cohorts of patients. Therefore, we performed a large, retrospective, matched analysis to evaluate the treatment outcomes of IC/CCRT and IC/RT in locoregionally advanced NPC.

\section{RESULTS}

\section{Baseline characteristics and treatment}

The clinical data of the 877 patients with advanced NPC treated between January 2004 and December 2010 who met all of the criteria was registered. After matching based on eight clinicopathological characteristics, a total of 642 patients were included in this study: 321 received IC followed by RT alone and 321 received IC plus CCRT. The median patient age was relatively low (50 years-old, range 15 to 70 years-old), 498/642 (77.6\%) patients were male and $144 / 642(22.4 \%)$ patients were female; 389/642 (60.6\%) patients had stage III NPC, 153 /642 (23.8\%), stage IVa, and 100/642 (15.6\%), stage IVb. Baseline characteristics were well balanced between the two arms (Table 1).

All 642 patients completed the prescribed course of two-dimensional conventional radiotherapy (2D-CRT). Of the 642 patients receiving IC, 128 patients completed one cycle of IC, 472 completed two cycles and 42 completed three or more cycles; 466/642 (72.6\%) received the PF regimen and 115/642 (17.9\%) received the PT regimen. In the IC/CCRT arm, 321 patients received concurrent chemotherapy; 58/321 (18\%) received weekly administration of cisplatin and 263/321 (82\%) received three courses of cisplatin, the median total dose of cisplatin was $240 \mathrm{mg} / \mathrm{m}^{2}$ (IQR 120-360). Overall, 102/321 (32\%) patients could not complete three courses of concurrent chemotherapy during RT due to toxic effects.

\section{Response and survival outcomes}

Three months after the completion of all treatment, treatment response was assessed for all 642 patients. The addition of concurrent chemotherapy to RT did not significantly increase the objective response rate (complete response and partial response; 97\% vs. 97\%, $P=1.000$ ).

The median follow-up duration for all patients was 73 months (range, 2-129 months). In the unmatched patients $(n=877)$, the 5-year OS, DFS, DMFS and LRRFS rates did not differ significantly between the IC/ CCRT and IC/RT arms (OS, 77\% vs. 75\%, $P=0.148$; DFS, $72 \%$ vs. $70 \%, P=0.355$; DMFS, $84 \%$ vs. $83 \%$, $P=0.949$; LRRFS, $89 \%$ vs $91 \%, P=0.267)$. In the matched patients $(n=642)$, the 5 -year overall survival rates for the IC/CCRT and IC/RT arms were $76 \%$ and $75 \%$, respectively, with a hazard ratio of $0.72(95 \% \mathrm{CI}$, 0.94 to 1.02). The corresponding 5-year DFS, DMFS, and LRRFS rates were $70 \%, 86 \%$, and $88 \%$ for the IC/CCRT arm and $70 \%, 90 \%$, and $91 \%$ for the IC/RT arm. Table 2 summarizes the survival outcomes of the two arms, and demonstrates the non-significant trends in favor of the addition of concurrent chemotherapy to IC/RT (Figure 1).

\section{Subgroup analysis}

Subgroup analyses were performed for T3-4 category, N2-3 category, IC regimen (PF or PT), and the number of IC cycles ( $\leq 2$ or $>2$ cycles). In the N2-3 category subgroup, concurrent chemotherapy seemed to provide an OS with a HR of 1.14 (95\% CI: 0.81-1.60), however, this trend did not reach statistical significance $(P$ $=0.465)$. In patients that received different IC regimens, concurrent chemotherapy tended to improve DFS, DMFS and LRRFS, though these effects were also not statistically significant $(P>0.05)$. Similarly, in the subgroup of IC cycles, no significant increase in any survival outcome 
Table 1: Clinicopathological characteristics of nasopharyngeal carcinoma patients with stage III-IVb

\begin{tabular}{|c|c|c|c|c|c|c|}
\hline & \multicolumn{3}{|c|}{ Unmatched } & \multicolumn{3}{|c|}{ Matched } \\
\hline & IC/CCRT & IC/RT & $P$-value & IC/CCRT & IC/RT & $P$-value \\
\hline & 499 (56.9\%) & $378(43.1 \%)$ & & $321(50.0 \%)$ & $321(50.0 \%)$ & \\
\hline Age (years) & & & 0.059 & & & 0.269 \\
\hline$\leq 45$ years & $262(52.5 \%)$ & $174(46.0 \%)$ & & $151(47.1 \%)$ & $165(51.4 \%)$ & \\
\hline$>45$ years & $237(47.5) \%$ & $204(54.0 \%)$ & & $170(52.9 \%)$ & $156(48.6 \%)$ & \\
\hline Sex & & & 0.696 & & & 0.256 \\
\hline Male & $378(75.8 \%)$ & $282(74.6 \%)$ & & $255(79.4 \%)$ & $243(75.7 \%)$ & \\
\hline Female & $121(24.2 \%)$ & $96(25.4 \%)$ & & $66(20.6 \%)$ & $78(24.3 \%)$ & \\
\hline $\mathrm{T}$ category & & & 0.720 & & & 0.819 \\
\hline $\mathrm{T} 1$ & $15(3.0 \%)$ & $11(2.9 \%)$ & & $13(4.0 \%)$ & $9(2.8 \%)$ & \\
\hline $\mathrm{T} 2$ & $75(15.0 \%)$ & $67(17.7 \%)$ & & $56(17.4 \%)$ & $59(18.4 \%)$ & \\
\hline $\mathrm{T} 3$ & $283(56.7 \%)$ & $203(53.7 \%)$ & & $167(52.0 \%)$ & $164(51.1 \%)$ & \\
\hline $\mathrm{T} 4$ & $126(25.3 \%)$ & $97(25.7 \%)$ & & $85(26.5 \%)$ & $89(27.7 \%)$ & \\
\hline $\mathrm{N}$ category & & & 0.006 & & & 0.438 \\
\hline No & $59(11.8 \%)$ & $35(9.3 \%)$ & & $24(7.5 \%)$ & $25(7.8 \%)$ & \\
\hline N1 & $174(34.9 \%)$ & $143(37.8 \%)$ & & $106(33.0 \%)$ & $114(35.5 \%)$ & \\
\hline $\mathrm{N} 2$ & $169(33.9 \%)$ & $155(41.0 \%)$ & & $134(41.7 \%)$ & $140(43.6 \%)$ & \\
\hline N3 & $97(19.4 \%)$ & $45(11.9 \%)$ & & $57(17.8 \%)$ & $42(13.1 \%)$ & \\
\hline Clinical stage & & & 0.011 & & & 0.213 \\
\hline III & $298(59.7 \%)$ & $245(64.8 \%)$ & & $190(59.2 \%)$ & $199(62.0 \%)$ & \\
\hline $\mathrm{IVa}$ & $104(20.8 \%)$ & $88(23.4 \%)$ & & $74(23.1 \%)$ & $80(24.9 \%)$ & \\
\hline $\mathrm{IVb}$ & $97(19.4 \%)$ & $45(11.9 \%)$ & & $57(17.8 \%)$ & $42(13.1 \%)$ & \\
\hline Histological type & & & 1.000 & & & 1.000 \\
\hline I & $0(0 \%)$ & $0(0 \%)$ & & $0(0 \%)$ & $0(0 \%)$ & \\
\hline II/III & $499(100 \%)$ & $378(100 \%)$ & & $321(100 \%)$ & $321(100 \%)$ & \\
\hline IC regimen & & & 0.145 & & & 0.130 \\
\hline Cisplatin/fluorouracil & $360(72.1 \%)$ & $279(73.8 \%)$ & & $228(71.0 \%)$ & $238(74.1 \%)$ & \\
\hline Cisplatin/paclitaxel & $94(18.8 \%)$ & $55(14.5 \%)$ & & $67(20.9 \%)$ & $48(15.0 \%)$ & \\
\hline Other & $45(9.0 \%)$ & $44(11.6 \%)$ & & $26(8.1 \%)$ & $35(10.9 \%)$ & \\
\hline IC cycles & & & 0.455 & & & 1.000 \\
\hline$\leq 2$ cycles & $472(94.6 \%)$ & $353(93.4 \%)$ & & $300(93.5 \%)$ & $300(93.5 \%)$ & \\
\hline$>2$ cycles & $27(5.4 \%)$ & $25(6.6 \%)$ & & $21(6.5 \%)$ & $21(6.5 \%)$ & \\
\hline
\end{tabular}

IC, induction chemotherapy; CCRT, concurrent chemoradiotherapy; RT, radiotherapy.

$P$-values were calculated using the Chi-square test, or Fisher's exact test if the expected number was $<5$ in at least $25 \%$ of cells. 
Table 2: Comparison of the survival rates for IC/CCRT versus IC/RT

\begin{tabular}{|c|c|c|c|c|}
\hline Variable & $\begin{array}{l}\text { IC/CCRT } \\
(n=321)\end{array}$ & $\begin{array}{l}\text { IC/RT } \\
\left(n^{-}=321\right)\end{array}$ & HR (95\% CI) & $P$-value \\
\hline Overall survival (\%) & & & $0.85(0.64-1.14)$ & 0.280 \\
\hline At 3-years & 83 & 82 & & \\
\hline At 5 -years & 76 & 75 & & \\
\hline Disease-free survival (\%) & & & $0.92(0.70-1.21)$ & 0.557 \\
\hline At 3-years & 73 & 74 & & \\
\hline At 5-years & 70 & 70 & & \\
\hline Distant metastasis-free survival (\%) & & & $1.41(0.85-2.35)$ & 0.188 \\
\hline At 3-years & 88 & 88 & & \\
\hline At 5-years & 86 & 90 & & \\
\hline Locoregional relapse-free survival (\%) & & & $0.91(0.61-1.36)$ & 0.650 \\
\hline At 3-years & 90 & 93 & & \\
\hline At 5-years & 88 & 91 & & \\
\hline
\end{tabular}

HR, hazard ratio; CI, confidence interval; IC, induction chemotherapy; CCRT, concurrent chemoradiotherapy; RT: radiotherapy.

Table 3: Subgroup analysis of IC/CCRT versus IC/RT by tumor stage and induction chemotherapy regimen

\begin{tabular}{|c|c|c|c|c|c|c|c|c|}
\hline \multirow[t]{2}{*}{ Subgroup } & \multicolumn{2}{|l|}{ Overall survival } & \multicolumn{2}{|c|}{ Disease-free survival } & \multicolumn{2}{|c|}{ Distant metastasis-free } & \multicolumn{2}{|c|}{ Locoregional relapse-free } \\
\hline & HR (95\% CI) & $P$ & HR (95\% CI) & $P$ & HR $(95 \%$ CI $)$ & $P$ & HR (95\% CI) & $P$ \\
\hline \multicolumn{9}{|l|}{ T, N stage } \\
\hline T3-4 & $0.91(0.65-1.26)$ & 0.556 & $0.92(0.67-1.26)$ & 0.583 & $1.00(0.64-1.58)$ & 0.996 & $1.03(0.54-1.99)$ & 0.921 \\
\hline $\mathrm{N} 2-3$ & $1.14(0.81-1.60)$ & 0.462 & $1.23(0.88-1.71)$ & 0.233 & $1.33(0.82-2.18)$ & 0.254 & $1.68(0.91-3.12)$ & 0.100 \\
\hline \multicolumn{9}{|l|}{ IC regimen } \\
\hline $\mathrm{PF}$ & $0.59(0.30-1.15)$ & 0.121 & $0.56(0.29-1.07)$ & 0.079 & $0,52(0.20-1.31)$ & 0.165 & $0.51(0.15-1.66)$ & 0.259 \\
\hline PT & $0.93(0.67-1.30)$ & 0.675 & $1.02(0.74-1.40)$ & 0.911 & $1.01(0.63-1.61)$ & 0.973 & $1.68(0.90-3.15)$ & 0.105 \\
\hline \multicolumn{9}{|l|}{ IC cycles } \\
\hline$\leq 2$ cycles & $0.85(0.63-1.15)$ & 0.284 & $0.91(0.69-1.21)$ & 0.528 & $0.91(0.60-1.37)$ & 0.649 & $1.450 .85-2.46()$ & 0.172 \\
\hline$>2$ cycles & $0.93(0.33-2.65)$ & 0.893 & $0.83(0.33-2.65)$ & 0.893 & $1,44(0.24-8.60)$ & 0.692 & $0.46(0.04-5.09)$ & 0.528 \\
\hline
\end{tabular}

HR, hazard ratio; CI, confidence interval; PF: cisplatin/fluorouracil; PT: cisplatin/paclitaxel; IC, induction chemotherapy.

was observed for patients who received concurrent chemotherapy $(P>0.05$, Table 3$)$.

\section{Prognostic factors}

Multivariate analysis was performed to further assess prognostic factors. Various potential prognostic factors for OS, DFS, DMFS and LRRFS, including sex, age, $\mathrm{T}$ category, $\mathrm{N}$ category, and treatment arm were evaluated. In multivariate analysis, $\mathrm{T}$ and $\mathrm{N}$ category were identified as significant prognostic factors for OS, DFS, DMFS, and age was an independent prognostic factor for OS (Table 4). Sex, age and treatment arm were not significant prognostic factors for any survival outcome. 
Table 4: Multivariate analysis of the associations between significant prognostic factors and survival outcomes in the propensity-matched cohort

\begin{tabular}{|c|c|c|c|}
\hline Outcome & Hazard ratio & $95 \% \mathrm{CI}$ & $P$-value \\
\hline \multicolumn{4}{|l|}{ Overall survival } \\
\hline Sex female vs. male & 0.73 & $0.50-1.06$ & 0.098 \\
\hline Age $>45$ vs. $\leq 45$ years & 1.47 & $1.10-1.97$ & 0.009 \\
\hline T category T1-2 vs.T3-4 & 1.50 & $1.23-1.82$ & 0.000 \\
\hline $\mathrm{N}$ category $\mathrm{N} 0-1$ vs. $\mathrm{N} 2-3$ & 1.72 & $1.43-2.08$ & 0.000 \\
\hline Treatment arm IC/CCRT vs. IC/RT & 0.87 & $0.65-1.16$ & 0.331 \\
\hline \multicolumn{4}{|l|}{ Distant-free survival } \\
\hline Sex female vs. male & 0.70 & $0.48-1.00$ & 0.051 \\
\hline Age $>45$ vs. $\leq 45$ years & 1.30 & $0.99-1.71$ & 0.064 \\
\hline T category T1-2 vs.T3-4 & 1.38 & $1.14-1.67$ & 0.001 \\
\hline $\mathrm{N}$ category $\mathrm{N} 0-1$ vs. $\mathrm{N} 2-3$ & 1.62 & $1.36-1.93$ & 0.000 \\
\hline Treatment arm IC/CCRT vs. IC/RT & 0.93 & $0.71-1.22$ & 0.597 \\
\hline \multicolumn{4}{|l|}{ Distant metastasis-free survival } \\
\hline Sex female vs. male & 0.60 & $0.34-1.06$ & 0.080 \\
\hline Age $>45$ vs. $\leq 45$ years & 1.38 & $0.92-2.08$ & 0.119 \\
\hline T category T1-2 vs.T3-4 & 1.51 & $1.14-2.00$ & 0.004 \\
\hline $\mathrm{N}$ category $\mathrm{N} 0-1$ vs. $\mathrm{N} 2-3$ & 1.82 & $1.40-2.38$ & 0.000 \\
\hline Treatment arm IC/CCRT vs. IC/RT & 0.92 & $0.62-1.38$ & 0.688 \\
\hline \multicolumn{4}{|l|}{ Locoregional relapse-free survival } \\
\hline Sex female vs. male & 0.74 & $0.39-1.43$ & 0.373 \\
\hline Age $>45$ vs. $\leq 45$ years & 0.93 & $0.56-1.54$ & 0.785 \\
\hline T category T1-2 vs.T3-4 & 0.86 & $0.61-1.22$ & 0.398 \\
\hline $\mathrm{N}$ category $\mathrm{N} 0-1$ vs. $\mathrm{N} 2-3$ & 1.25 & $0.89-1.75$ & 0.193 \\
\hline Treatment arm IC/CCRT vs. IC/RT & 1.36 & $0.82-2,27$ & 0.238 \\
\hline
\end{tabular}

IC, induction chemotherapy; CCRT, concurrent chemoradiotherapy; RT: radiotherapy.

$P$-values were calculated using the Chi-square test (or Fisher's exact test if the expected number was $<5$ in at least $25 \%$ of cells).

\section{Toxicities}

No treatment-related deaths were observed in either arm. Overall, in terms of acute toxicities during IC, we noted no significant differences between the two arms $(P$ $>0.05)$. Regarding acute toxicities during RT, grade 3-4 adverse events appeared to be more frequent in the IC/ CCRT arm than the IC/RT arm. For example, the most common gastrointestinal events were nausea/vomiting (24\% vs. $3 \%, P=0.000)$ and mucositis $(49 \%$ vs. $30 \%, P$ $=0.000)$, and the most frequent hematological toxicities were leucopenia/neutropenia ( $12 \%$ vs. $1.9 \%, P=0.000)$, thrombocytopenia ( $9 \%$ vs. $1.2 \%, P=0.000)$ and anemia (3.7\% vs. $0.6 \%, P=0.015)$. In analysis of grade 3-4 late toxicities, the frequencies of cranial neuropathy, temporal lobe necrosis, ear problems (deafness/otitis) and neck tissue damage were similar between the IC/CCRT and IC/ RT arms $(P>0.05$; Table 5).

\section{DISCUSSION}

This retrospective matched analysis assessed a relatively large cohort of patients with locally advanced NPC $(n=642)$ in order to further compare the survival outcomes and toxicities of IC followed by RT alone or CCRT. Although IC followed by CCRT has emerged as the current standard treatment for locoregionally advanced NPC according to the NCCN guidelines [2], the present study indicates the addition of concurrent chemotherapy does not translate to any significant survival advantage 
over IC/RT. Moreover, IC/CCRT appears to increase the risk of toxicities.

Both induction and concurrent chemotherapy have been proposed as effective treatment strategies for NPC [12-14]. However, the most beneficial sequence of chemotherapy for patients has not yet been established. Lin et al. reported the advantages of IC plus RT in terms of 3-year local control and overall survival $(95 \%, 89 \%)$ in NPC [15]. Additionally, a meta-analysis demonstrated $\mathrm{IC}$ followed by $\mathrm{RT}$ reduced the locoregional recurrence rate (LRR) and distant metastasis rate (DMR) and improved overall survival (OS) in NPC [16]. In a longterm outcome report [17], IC followed by RT resulted in excellent 10-year overall survival and failure-free survival rates of $49.5 \%$ and $48 \%$. In the current study, IC plus RT also provided superb outcomes in terms of 5-year overall survival $(75 \%)$ and disease-free survival $(70 \%)$ in patients with locoregionally advanced NPC.

A prior study conducted by Huang et al. [6] administered chemotherapy concurrently with radiotherapy after IC in the hope of achieving survival

A

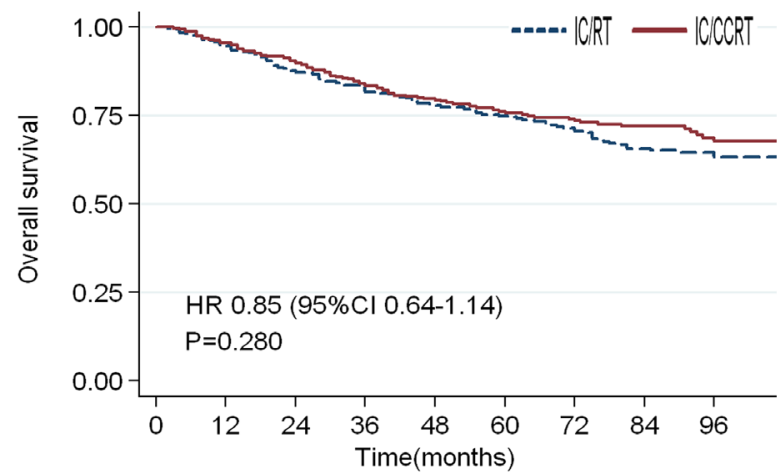

Number at risk

IC/RT $321 \quad 300 \quad 270 \quad 247 \quad 224 \quad 201 \quad 176 \quad 130 \quad 53$ $\begin{array}{llllllllll}\text { IC/CCRT } 321 & 302 & 280 & 258 & 236 & 194 & 153 & 115 & 67\end{array}$

C

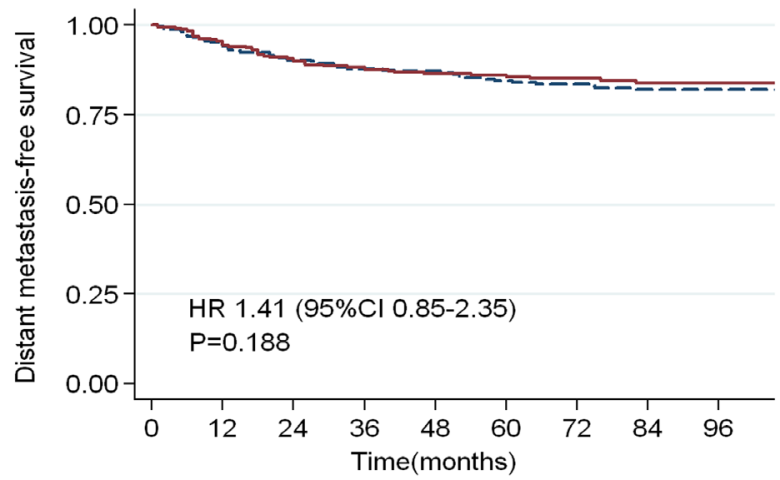

Number at risk

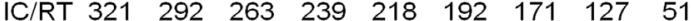

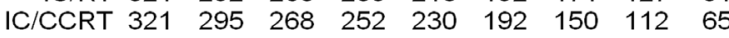

benefits. Unfortunately, the addition of concurrent chemotherapy did not result in superior survival outcomes compared to IC/RT. Indeed, several trials have reported the same outcomes for IC/RT and IC/CCRT. The small retrospective study conducted by $\mathrm{Li}$ et al. demonstrated that IC/CCRT did not provide any survival benefit over IC/RT $(P>0.05)[11]$. In the trial conducted by Su et al., additional concurrent chemotherapy did not provide a significant OS benefit over IC/RT (82.3\% vs. 73.4\%, $P>$ 0.05 ) in locally advanced NPC [18]. In this study, we also failed to observe a better outcome for IC/CCRT over IC/RT in terms of either 5-year OS $(76 \% v s .75 \%, P=0.280)$ or DFS (70\% vs. $70 \%, P=0.557)$. Evaluation of prognostic factors using multivariable analysis demonstrated concurrent chemotherapy was also not an independent factor affecting survival. Furthermore, subgroup analysis did not indicate significant prognostic value for concurrent chemotherapy. Therefore, the available evidence indicates additional concurrent chemotherapy negatively affects treatment, rather than providing a survival benefit.

Compared to concurrent chemotherapy, IC has

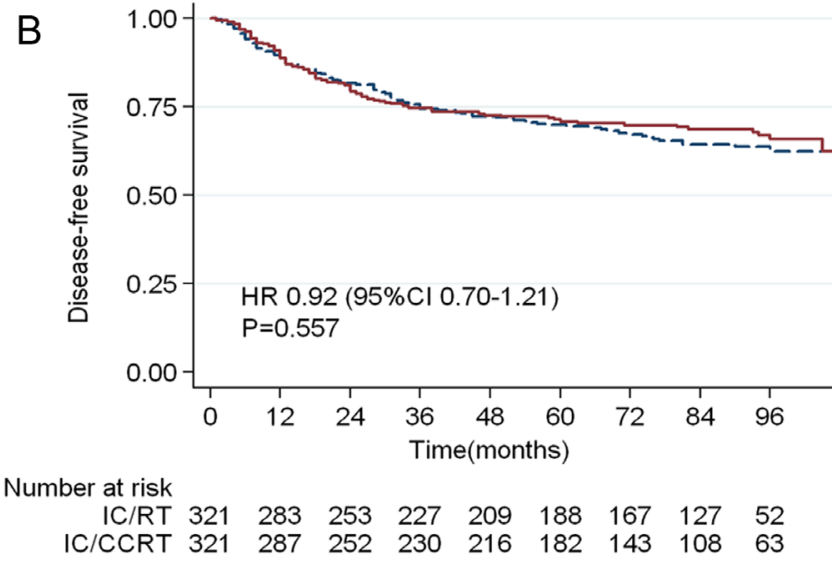

D

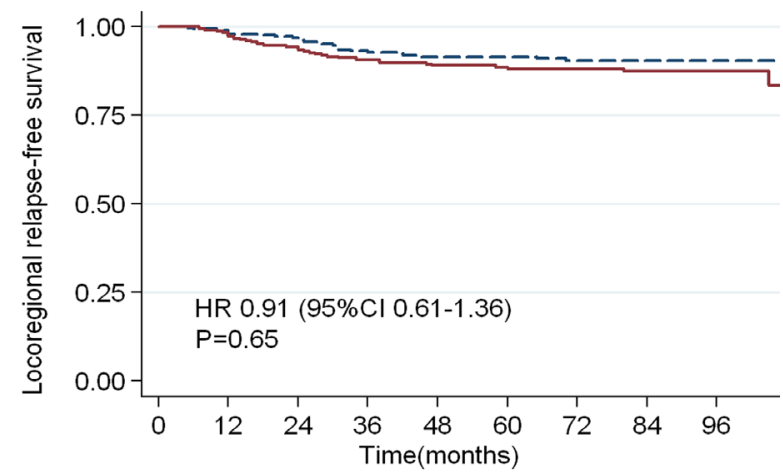

Number at risk

IC/RT $321 \quad 298 \quad 263 \quad 234 \quad 214 \quad 196 \quad 172 \quad 129 \quad 53$

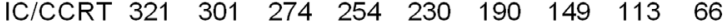

Figure 1: Kaplan-Meier survival curves for patients with locoregionally advanced nasopharyngeal carcinoma in the IC/CCRT and IC/RT arms. A. overall survival; B. disease-free survival; C. distant metastasis-free survival; D. locoregional relapsefree survival. 
Table 5: Profile of treatment-related toxicities

\begin{tabular}{|c|c|c|c|}
\hline & IC/CCRT $(n=321)$ & IC/RT $(n=321)$ & $P$ \\
\hline \multicolumn{4}{|c|}{ Grade 3-4 adverse events during IC, $n(\%)$} \\
\hline \multicolumn{4}{|l|}{ Hematological } \\
\hline Leukopenia/neutropenia & $100(31 \%)$ & $106(33 \%)$ & 0.672 \\
\hline Thrombocytopenia & $16(5 \%)$ & $13(4 \%)$ & 0.704 \\
\hline Anemia & $13(4 \%)$ & $10(3 \%)$ & 0.671 \\
\hline \multicolumn{4}{|l|}{ Non-hematological } \\
\hline Stomatitis (mucositis) & $5(1.6 \%)$ & $3(1 \%)$ & 0.722 \\
\hline Nausea/ vomiting & $55(17 \%)$ & $48(15 \%)$ & 0.519 \\
\hline Diarrhea & $8(2.5 \%)$ & $11(3.5 \%)$ & 0.641 \\
\hline Liver dysfunction & $3(1 \%)$ & $2(0.6 \%)$ & 1.000 \\
\hline Kidney dysfunction & 0 & 0 & 1.000 \\
\hline \multicolumn{4}{|c|}{ Grade 3-4 adverse events during RT, $n(\%)$} \\
\hline \multicolumn{4}{|l|}{ Hematological } \\
\hline Leukopenia/neutropenia & $39(12 \%)$ & $6(1.9 \%)$ & 0.000 \\
\hline Thrombocytopenia & $28(9 \%)$ & $4(1.2 \%)$ & 0.000 \\
\hline Anemia & $12(3.7 \%)$ & $2(0.6 \%)$ & 0.015 \\
\hline \multicolumn{4}{|l|}{ Non-hematological } \\
\hline Skin reaction (radiation-related) & $22(7 \%)$ & $16(5 \%)$ & 0.403 \\
\hline Mucositis (radiation-related) & $157(49 \%)$ & $96(30 \%)$ & 0.000 \\
\hline Nausea /vomiting & $77(24 \%)$ & $9(3 \%)$ & 0.000 \\
\hline Dry mouth & $102(32 \%)$ & $87(27 \%)$ & 0.225 \\
\hline \multicolumn{4}{|l|}{ Grade 3-4 late toxicities, $n(\%)$} \\
\hline Cranial neuropathy & $14(4.5 \%)$ & $16(5 \%)$ & 0.708 \\
\hline Temporal lobe necrosis & $22(7 \%)$ & $29(9 \%)$ & 0.307 \\
\hline Ear (deafness/otitis) & $132(41 \%)$ & $119(37 \%)$ & 0.293 \\
\hline Neck tissue damage & $80(25 \%)$ & $67(21 \%)$ & 0.222 \\
\hline
\end{tabular}

IC, induction chemotherapy; CCRT, concurrent chemoradiotherapy; RT: radiotherapy; CI, confidence interval.

several potential advantages. Firstly, early use of chemotherapy drugs at full dose before RT enables better drug delivery through the vasculature and can effectively eradicate micrometastases. Secondly, IC can be better tolerated by patients at the initial stage of treatment, which leads to better compliance. Finally, IC is administered to shrink the primary tumor and increase tumor radiosensitivity $[19,20]$. Simultaneously, a favorable increase in locoregional control is achieved with concurrent chemotherapy; however, its ability to control distant metastasis is relatively limited, as proven in numerous studies [21, 22]. Additionally, with the development of imaging techniques, MRI, 18F-FDG PET/CT and
HRCT are recommended as the preferred modalities for staging patients with NPC $[9,10]$. Likewise, the gross tumor volume (GTV) is defined more accurately during radiotherapy planning for 2D-CRT, which provides significant value in terms of locoregional control in NPC. Notably, further improvement in locoregional control by the addition of concurrent chemotherapy is meaningless. Therefore, based on our results, we do not recommend the addition of concurrent chemotherapy to RT after IC in locally advanced NPC.

Existing data indicates drug-related toxicities induced by CCRT lead to the interruption of RT or concurrent chemotherapy in many patients with NPC 
$[23,24]$. The current study reveals additional concurrent chemotherapy increases the frequency of grade 3-4 toxicities, especially vomiting, nausea, leukopenia, thrombocytopenia and anemia, in agreement with the results of a phase III randomized trial reported by Huang et al. [6]. Due to these excess toxicities, the tolerance of patients to concurrent chemotherapy during RT was usually very poor and compliance was $68 \%$ in this study, in accordance with other studies [25].

There are several limitations to this study. First, this was a retrospective analysis; therefore, selection bias could not be avoided, although pair-matched analysis can reduce selection bias. Secondly, standardized protocols and chemotherapy strategies were not employed for the entire cohort. Thirdly, 2D-CRT was used as the radiation technology; this technique is proven to result in a lower locoregional control rate than IMRT [26]. Therefore, the results of this study require validation in phase III prospective trials of patients with locally advanced NPC treated using IMRT.

In conclusion, this analysis failed to demonstrate the superiority of IC/CCRT over IC/RT in locoregionally advanced NPC, with concurrent chemotherapy contributing to higher frequencies of toxicities. In light of the wide-spread adoption of advanced RT technologies, we plan to conduct a prospective trial address the true value of IC/CCRT versus IC/RT in patients with locally advanced NPC treated using IMRT.

\section{MATERIALS AND METHODS}

\section{Participants and study design}

A total of 1697 NPC patients who received IC between January 2004 and December 2010 were retrospectively accrued from four radiation oncology institutions: the Affiliated Hospital of Guilin Medical University, Wuzhou Red Cross Hospital, 181st Hospital of People's Liberation Army, and Nanxishan Hospital. The inclusion criteria were newly pathologically-diagnosed, non-distant metastatic stage III-IVb NPC according to the 7 th edition of American Joint Committee on Cancer (AJCC) or Union for International Cancer Control (UICC) staging system [27]; age 15-70 years-old; and normal complete blood count, normal hepatic and renal function and a Karnofsky Score $\geq 70$. The exclusion criteria were: age $>70$ years-old $(n=9)$, stage I or stage II NPC $(n=$ $151)$, patients who received adjuvant chemotherapy ( $n=$ $584)$ and those with insufficient data $(n=76)$. Ultimately, 877 patients with locoregionally advanced NPC who had been treated with IC/RT or IC/CCRT were included. The two arms were matched for eight clinicopathological characteristics [28]: age ( $\leq 45$ years $v s .>45$ years), sex (male vs. female), T category (T1 vs. T2 vs. T3 vs. T4), $\mathrm{N}$ category (N0vs. N1 vs. N2 vs. N3), clinical stage (III vs. IVa $v s$. IVb), histological type (type I vs. type II/III), IC regimen (cisplatin/fluorouracil vs. cisplatin/paclitaxel vs. other) and number of IC cycles ( $\leq 2 v s .>2$ cycles). After matching, 642 patients were classified into one of the two arms: IC followed by RT or IC followed by CCRT, with 321 patients in each arm. Overall survival (OS), diseasefree survival (DFS), distant metastasis-free survival (DMFS), locoregional relapse-free survival (LRRFS) and treatment related toxicities were compared between the two arms.

The study was conducted in accordance with the Declaration of Helsinki, Good Clinical Practice Guidelines and national and international guidelines, and was approved by the Research Ethics Committees of all four institutions. All patients provided written informed consent.

\section{Treatment}

All patients underwent two-dimensional conventional radiotherapy (2D-CRT) delivered by a linear accelerator device including 6 million Volt photons. The primary nasopharyngeal tumor was irradiated with a dose of 68-74 Gy. The metastatic lymph nodes in the cervical region were prescribed a dose of 66-70 Gy and the cervical region without lymph nodes metastases a dose of 50-54 Gy. All patients were treated with 2.0 Gy per fraction daily, 5 days per week. During the period of IC, 466 patients received cisplatin $\left(25 \mathrm{mg} / \mathrm{m}^{2}\right.$, on day $\left.1-3\right)$ and 5-fluorouracil (600-800 mg/m², on days $1-5), 115$ patients received cisplatin $\left(25 \mathrm{mg} / \mathrm{m}^{2}\right.$, on day $\left.1-3\right)$ and paclitaxel $\left(135 \mathrm{mg} / \mathrm{m}^{2}\right.$, on day 1$)$, and 61 patients received other regimens (such as cisplatin plus docetaxel or cisplatin plus gemcitabine). All regimens were repeated every 3 weeks for one or more cycles. After IC, all patients were treated with 2D-CRT. The study-defined CCRT regimen was 30 $\mathrm{mg} / \mathrm{m}^{2}$ cisplatin on day 1 per week for 6 cycles or $75 \mathrm{mg} /$ $\mathrm{m}^{2}$ cisplatin on day 1 every 3 weeks for 3 cycles.

\section{Assessments and follow up}

Pretreatment assessments included a physical examination, chest CT, electrocardiogram, liver ultrasound, dental examination, and other laboratory investigations such as a complete blood cell count and full biochemical profiles. Nasopharyngeal fiber optic endoscopy and biopsy, and MRI or CT of the nasopharynx and neck were performed at baseline. Patients with bone pain underwent chest and abdominal CT scans and bone ECT scans for metastatic workup.

Clinical evaluation of efficacy of treatment was assessed according to the Response Evaluation Criteria in Solid Tumors (RECIST) version 1.0 [29]. Acute treatment-related toxicities (occurring from the start of 
IC to three months after RT) were evaluated based on the Common Terminology Criteria for Adverse Events (CTCAE) version 3.0 [30]. Late RT-related toxicities (occurring or persisting beyond three months from initiation of RT) were graded according to the Radiation Morbidity Scoring Criteria of the Radiation Therapy Oncology Group. Follow-up was measured from the first day of treatment to the last day of examination or death. During the follow-up period, endoscopic biopsy and MRI or CT of the nasopharynx and neck were performed to diagnose all locoregional recurrences and irresolute cases were diagnosed by fine-needle aspiration. Distant metastasis was confirmed by chest and liver CT scans or bone emission computed tomography scans. All patients were assessed every 3 months for the first 3 years, every 6 months in the fourth and fifth years and annually thereafter.

\section{Endpoints and statistical analysis}

The primary endpoint was overall survival (OS, from initiation of treatment to death of any cause or last follow-up). Secondary clinical endpoints included disease-free survival (DFS, from initiation of treatment to first disease progression [local recurrence and/or distant metastasis] or death from any cause), distant metastasisfree survival (DMFS, from initiation of treatment to first distant metastasis), locoregional relapse-free survival (LRRFS, from initiation of treatment to locoregional progression), and treatment-related toxicities.

Clinical characteristics and treatment-related toxicity rates were evaluated using Fisher's exact test. The Kaplan-Meier method was used to estimate survival rates and the log-rank test was used to compare the survival distributions. Cox proportional hazards model was employed to identify prognostic factors associated with survival outcomes and estimate hazard ratios (HR) using the backward stepwise method. The potentially significant prognostic factors included the following: sex, age, T category, $\mathrm{N}$ category and treatment strategies. All statistical analyses were carried out using Stata version 13.0 (StataCorp LP, College Station, Texas, USA). Twotailed $P$-values $<0.05$ were considered statistically significant.

\section{CONFLICTS OF INTEREST}

The authors have no potential conflicts of interest to disclose.

\section{GRANT SUPPORT}

This work was supported by grants from the Scientific Research and Technology Development Program of Guilin (No. 20110119-1-3), the National
Natural Science Foundation of China (No. 81560443), the Guangxi National Natural Science Foundation (No.2013GXNSFBA019155), and the Guangxi Medical Scientific Experiment Center Open Fund (GK2014TKF04), the Guangxi Zhuang Autonomous Region Health and Family Planning Commission Fund (Z2016820 and Z2015395).

\section{REFERENCES}

1. Wei WI and Sham JS. Nasopharyngeal carcinoma. Lancet. 2005; 365:2041-2054.

2. National Comprehensive Cancer Network. NCCN Guidelines $^{\circledR}$. Available from http://www.nccn.org/ professionals/physician_gls/f_guidelines.asp. Accessed July 9, 2015.

3. Hui EP, Ma BB, Leung SF, King AD, Mo F, Kam MK, Yu BK, Chiu SK, Kwan WH, Ho R, Chan I, Ahuja AT, Zee $\mathrm{BC}$ and Chan AT. Randomized phase II trial of concurrent cisplatin-radiotherapy with or without neoadjuvant docetaxel and cisplatin in advanced nasopharyngeal carcinoma. J Clin Oncol. 2009; 27:242-249.

4. Kong L, Hu C, Niu X, Zhang Y, Guo Y, Tham IW and Lu JJ. Neoadjuvant chemotherapy followed by concurrent chemoradiation for locoregionally advanced nasopharyngeal carcinoma: interim results from 2 prospective phase 2 clinical trials. Cancer. 2013; 119:4111-4118.

5. Lee N, Harris J, Garden AS, Straube W, Glisson B, Xia P, Bosch W, Morrison WH, Quivey J, Thorstad W, Jones C and Ang KK. Intensity-modulated radiation therapy with or without chemotherapy for nasopharyngeal carcinoma: radiation therapy oncology group phase II trial 0225 . J Clin Oncol. 2009; 27:3684-3690.

6. Huang PY, Cao KJ, Guo X, Mo HY, Guo L, Xiang YQ, Deng MQ, Qiu F, Cao SM, Guo Y, Zhang L, Li NW, Sun R, Chen QY, Luo DH, Hua YJ, et al. A randomized trial of induction chemotherapy plus concurrent chemoradiotherapy versus induction chemotherapy plus radiotherapy for locoregionally advanced nasopharyngeal carcinoma. Oral Oncol. 2012; 48:1038-1044.

7. Zeng Q, Wang J, Lv X, Li J, Yin LJ, Xiang YQ and Guo $\mathrm{X}$. Induction Chemotherapy Followed by Radiotherapy versus Concurrent Chemoradiotherapy in elderly patients with nasopharyngeal carcinoma: finding from a propensitymatched analysis. BMC Cancer. 2016; 16:693.

8. Wu SY, Wu YH, Yang MW, Hsueh WT, Hsiao JR, Tsai ST, Chang KY, Chang JS and Yen CJ. Comparison of concurrent chemoradiotherapy versus neoadjuvant chemotherapy followed by radiation in patients with advanced nasopharyngeal carcinoma in endemic area: experience of 128 consecutive cases with 5 year follow-up. BMC Cancer. 2014; 14:787.

9. Abdel Khalek Abdel Razek A and King A. MRI and CT of nasopharyngeal carcinoma. AJR Am J Roentgenol. 2012; 198:11-18. 
10. Vellayappan BA, Soon YY, Earnest A, Zhang Q, Koh WY, Tham IW and Lee KM. Accuracy of (18) F-flurodeoxyglucose-positron emission tomography/ computed tomography in the staging of newly diagnosed nasopharyngeal carcinoma: a systematic review and metaanalysis. Radiol Oncol. 2014; 48:331-338.

11. Li WF, Li YQ, Chen L, Zhang Y, Guo R, Zhang F, Peng $\mathrm{H}$, Sun $\mathrm{Y}$ and Ma J. Propensity-matched analysis of three different chemotherapy sequences in patients with locoregionally advanced nasopharyngeal carcinoma treated using intensity-modulated radiotherapy. BMC Cancer. 2015; 15:810.

12. Langendijk JA, Leemans CR, Buter J, Berkhof J and Slotman BJ. The additional value of chemotherapy to radiotherapy in locally advanced nasopharyngeal carcinoma: a meta-analysis of the published literature. J Clin Oncol. 2004; 22:4604-4612.

13. Tan T, Lim WT, Fong KW, Cheah SL, Soong YL, Ang MK, Ng QS, Tan D, Ong WS, Tan SH, Yip C, Quah D, Soo $\mathrm{KC}$ and Wee J. Concurrent chemo-radiation with or without induction gemcitabine, Carboplatin, and Paclitaxel: a randomized, phase $2 / 3$ trial in locally advanced nasopharyngeal carcinoma. Int J Radiat Oncol Biol Phys. 2015; 91:952-960.

14. OuYang PY, Xie C, Mao YP, Zhang Y, Liang XX, Su Z, Liu Q and Xie FY. Significant efficacies of neoadjuvant and adjuvant chemotherapy for nasopharyngeal carcinoma by meta-analysis of published literature-based randomized, controlled trials. Ann Oncol. 2013; 24:2136-2146.

15. Lin S, Lu JJ, Han L, Chen Q and Pan J. Sequential chemotherapy and intensity-modulated radiation therapy in the management of locoregionally advanced nasopharyngeal carcinoma: experience of 370 consecutive cases. BMC Cancer. 2010; 10:39.

16. Chen YP, Guo R, Liu N, Liu X, Mao YP, Tang LL, Zhou GQ, Lin AH, Sun Y and Ma J. Efficacy of the Additional Neoadjuvant Chemotherapy to Concurrent Chemoradiotherapy for Patients with Locoregionally Advanced Nasopharyngeal Carcinoma: a Bayesian Network Meta-analysis of Randomized Controlled Trials. J Cancer. 2015; 6:883-892.

17. Huang PY, Zeng Q, Cao KJ, Guo X, Guo L, Mo HY, Wu PH, Qian CN, Mai HQ and Hong MH. Ten-year outcomes of a randomised trial for locoregionally advanced nasopharyngeal carcinoma: A single-institution experience from an endemic area. Eur J Cancer. 2015; 51:1760-1770.

18. Su SF, Han F, Zhao C, Huang Y, Chen CY, Xiao WW, Li JX and Lu TX. Treatment outcomes for different subgroups of nasopharyngeal carcinoma patients treated with intensitymodulated radiation therapy. Chin J Cancer. 2011; 30:565573.

19. Fountzilas G, Ciuleanu E, Bobos M, Kalogera-Fountzila A, Eleftheraki AG, Karayannopoulou G, Zaramboukas T, Nikolaou A, Markou K, Resiga L, Dionysopoulos D, Samantas E, Athanassiou H, Misailidou D, Skarlos D and Ciuleanu T. Induction chemotherapy followed by concomitant radiotherapy and weekly cisplatin versus the same concomitant chemoradiotherapy in patients with nasopharyngeal carcinoma: a randomized phase II study conducted by the Hellenic Cooperative Oncology Group (HeCOG) with biomarker evaluation. Ann Oncol. 2012; 23:427-435.

20. Kong L, Hu C, Niu X, Zhang Y, Guo Y, Tham IW and Lu JJ. Neoadjuvant chemotherapy followed by concurrent chemoradiation for locoregionally advanced nasopharyngeal carcinoma: interim results from 2 prospective phase 2 clinical trials. Cancer. 2013; 119:4111-4118.

21. Lee AW, Tung SY, Ngan RK, Chappell R, Chua DT, Lu TX, Siu L, Tan T, Chan LK, Ng WT, Leung TW, Fu YT, Au GK, Zhao C, O'Sullivan B, Tan EH, et al. Factors contributing to the efficacy of concurrent-adjuvant chemotherapy for locoregionally advanced nasopharyngeal carcinoma: combined analyses of NPC-9901 and NPC-9902 Trials. Eur J Cancer. 2011; 47:656-666.

22. Chua DT, Ma J, Sham JS, Mai HQ, Choy DT, Hong MH, Lu TX and Min HQ. Long-term survival after cisplatinbased induction chemotherapy and radiotherapy for nasopharyngeal carcinoma: a pooled data analysis of two phase III trials. J Clin Oncol. 2005; 23:1118-1124.

23. Chen Y, Sun Y, Liang SB, Zong JF, Li WF, Chen M, Chen L, Mao YP, Tang LL, Guo Y, Lin AH, Liu MZ and Ma J. Progress report of a randomized trial comparing long-term survival and late toxicity of concurrent chemoradiotherapy with adjuvant chemotherapy versus radiotherapy alone in patients with stage III to IVB nasopharyngeal carcinoma from endemic regions of China. Cancer. 2013; 119:22302238.

24. Ghi MG, Paccagnella A, D'Amanzo P, Mione CA, Fasan S, Paro S, Mastromauro C, Carnuccio R, Turcato G, Gatti C, Pallini A, Nascimben O, Biason R, Oniga F, Medici M, Rossi F, et al. Neoadjuvant docetaxel, cisplatin, 5-fluorouracil before concurrent chemoradiotherapy in locally advanced squamous cell carcinoma of the head and neck versus concomitant chemoradiotherapy: a phase II feasibility study. Int J Radiat Oncol Biol Phys. 2004; 59:481-487.

25. Fountzilas G, Ciuleanu E, Bobos M, Kalogera-Fountzila A, Eleftheraki AG, Karayannopoulou G, Zaramboukas T, Nikolaou A, Markou K, Resiga L, Dionysopoulos D, Samantas E, Athanassiou H, Misailidou D, Skarlos D and Ciuleanu $T$. Induction chemotherapy followed by concomitant radiotherapy and weekly cisplatin versus the same concomitant chemoradiotherapy in patients with nasopharyngeal carcinoma: a randomized phase II study conducted by the Hellenic Cooperative Oncology Group (HeCOG) with biomarker evaluation. Ann Oncol. 2012; 23:427-435.

26. Zhang MX, Li J, Shen GP, Zou X, Xu JJ, Jiang R, You R, Hua YJ, Sun Y, Ma J, Hong MH and Chen MY. Intensity-modulated radiotherapy prolongs the survival of 
patients with nasopharyngeal carcinoma compared with conventional two-dimensional radiotherapy: A 10-year experience with a large cohort and long follow-up. Eur J Cancer. 2015; 51:2587-2595.

27. Edge SB and Compton CC. The American Joint Committee on Cancer: the 7th edition of the AJCC cancer staging manual and the future of TNM. Ann Surg Oncol. 2010; 17:1471-1474.

28. Guo SS, Tang LQ, Chen QY, Zhang L, Liu LT, Guo L, Mo HY, Luo DH, Huang PY, Xiang YQ, Sun R, Chen MY, Wang L, Lv X, Zhao C, Guo X, et al. Induction chemotherapy followed by concurrent chemoradiotherapy versus concurrent chemoradiotherapy alone in stage III-IVb nasopharyngeal carcinoma patients with Epstein-Barr virus DNA $>/=4000$ copies/ml: a matched study. Oncotarget. 2016; 7:29739-29748. doi: 10.18632/oncotarget.8828.
29. Tsuchida Y and Therasse P. Response evaluation criteria in solid tumors (RECIST): new guidelines. Med Pediatr Oncol. 2001; 37:1-3.

30. Yoshida K, Yamazaki H, Nakamara S, Masui K, Kotsuma T, Akiyama H, Tanaka E and Yoshioka Y. Comparison of common terminology criteria for adverse events v3.0 and radiation therapy oncology group toxicity score system after high-dose-rate interstitial brachytherapy as monotherapy for prostate cancer. Anticancer Res. 2014; 34:2015-2018. 